\title{
A Frailty Assessment Tool to Predict In-Hospital Mortality in Patients with Acute Exacerbations of Chronic Obstructive Pulmonary Disease
}

Jin-Jin Gu $\mathbb{D}^{\prime}$
Qiang Liu $\mathbb{D}^{2}$
Li-Jie Zheng

'Department of Geriatrics, The Affiliated People's Hospital of Jiangsu University, Zhenjiang, 212002, People's Republic of China; ${ }^{2}$ Department of Laboratory Medicine, The Affiliated Hospital of Jiangsu University, Zhenjiang, 2I200I, People's Republic of China; ${ }^{3}$ Department of Laboratory Medicine,The Affiliated People's Hospital of Jiangsu University, Zhejiang, 2I 2002, People's Republic of China
Correspondence: Jin-Jin Gu Department of Geriatrics, The Affiliated People's Hospital of Jiangsu University, No. 8 Dianli Road, Zhenjiang, 212002, People's Republic of China Tel +86 5 II 8891542I

Email Gujj@jskfhn.org.cn
Background: The exacerbation of chronic obstructive pulmonary disease (AECOPD) is a chronic, frequent, and life-threatening lung disease. In 2014, a frailty index (FI) based on deficits in commonly used laboratory tests (FI-Lab) was suggested to identify older adults at increased risk of death.

Objective: We aim to study the prognostic value of the FI-Lab in older Chinese patients who were admitted because of AECOPD.

Methods: We screened 1932 older patients hospitalized with AECOPD from September 2016 to June 2019 at Zhenjiang First People's Hospital, China. A multivariate logistic regression analysis was used to identify prognostic factors for in-hospital mortality. Results: A total of 77 survivors and 77 non-survivors were finally included in the study. Both the mean DECAF (including dyspnea, eosinopenia, consolidation, acidemia, and atrial fibrillation) score and the mean FI-Lab value of non-survivors were statistically higher than those of survivors $(4.45 \pm 0.80$ versus $3.03 \pm 0.90, P=0.000 ; 0.51 \pm 0.13$ versus $0.29 \pm 0.10$, $P=0.000$, respectively). Logistic regression analysis suggested that DECAF Rank and FI-Lab Rank were strongly related factors of death in AECOPD patients. The areas under the receiver-operating characteristic (ROC) curves were 0.906 for FI-Lab and 0.870 for DECAF $(P=0.2991)$.

Conclusion: FI-Lab is a simple, efficient, and objective tool to stratify the risk of in-hospital mortality of AECOPD.

Keywords: frailty index, FI-Lab, DECAF, AECOPD, prognosis

\section{Introduction}

Chronic obstructive pulmonary disease (COPD) is a chronic, frequent, and lifethreatening lung disease. The acute exacerbation of chronic obstructive pulmonary disease (AECOPD) suggests decreased physical activity and pulmonary function. ${ }^{1}$ A simple prognosis tool can contribute to clinical management, early risk stratification, and prevention of poor outcomes, as well as monitoring during treatment. Clinicians are constantly seeking predictors of mortality for patients with AECOPD. Current prognostic markers for AECOPD include two main categories: inflammation-related markers ${ }^{2-4}$ and dyspnea, eosinopenia, consolidation, acidemia, and atrial fibrillation (DECAF) score. ${ }^{5-7}$

Inflammatory mediators can destroy lung structure and promote neutrophil inflammation, which can worsen illness and cause death. Inflammation-related markers such as C-reactive protein (CRP) and interleukin, ${ }^{2,4}$ red blood cell 
distribution (RDW) widths, ${ }^{8,9}$ eosinophil counts, ${ }^{10}$ and neutrophil and lymphocyte ratio (NLR) ${ }^{11}$ are often associated with mortality or readmission of AECOPD patients. $\operatorname{DECAF}^{5,7,12}$ (including dyspnea, eosinopenia, pulmonary consolidation, acidemia, and atrial fibrillation) is also a commonly used predictor of in-hospital mortality in AECOPD patients.

However, most of the inflammation-related markers can only suggest significant differences between patients with AECOPD and patients with stable COPD, while the accuracy of their prognoses is relatively low. The DECAF scoring system requires five indicators: clinical presentation, electrocardiogram, imaging (chest x-ray or computed tomography), blood gas analysis, and routine blood work. DECAF scoring requires relatively specialized knowledge. First, it is a little difficult to evaluate dyspnea and changes in lung consolidation. Moreover, DECAF scoring is not practical for repeated evaluations of patients because imaging changes are relatively slow. Therefore, no generally accepted indicator exists. We need objective predictions that are easy to obtain, to be able to provide more accurate prognoses regarding AECOPD patients, and perhaps we could try interdisciplinary indexes as well.

Frailty is a clinical condition characterized by a decline in reserves and resistance to stressors. It pervades older adults and causes them to be more vulnerable to numerous health problems, as well as at greater risk of dying. ${ }^{13,14}$ Older patients are now recommended for frailty assessments. ${ }^{15}$ In addition, people with COPD are more likely to coexist with frailty. Indeed, they share the same risk factors as do seniors and those who smoke. Common pathogenesis, including inflammatory lesions and endocrine dysfunction, could affect both COPD and frailty. ${ }^{16,17}$ Can an objective and easily measurable frailty index be found for predicting mortality in AECOPD patients?

The frailty index based on routine laboratory tests (FI$\mathrm{Lab}$ ) is a comprehensive, objective, and readily detectable index for quantifying frailty. The FI-Lab consists of 21 common blood tests (complete blood count, kidney function, thyroid function, liver function, electrolyte, etc.) plus systolic and diastolic blood pressures. ${ }^{18}$ An FI-Lab value is calculated by counting the number of deficits in a patient and dividing by the total number of deficits measured to produce a score between 0 and 1. A higher score indicates greater frailty. Although studies by Rockwood et $\mathrm{al}^{19}$ and Howlett et $\mathrm{al}^{20}$ have confirmed that the FI-Lab can quantify health and point to adverse outcomes including death, most of the pre-existing prognostic studies using the FI-Lab only focused on long-term care nursing homes. ${ }^{13,20,21}$ Therefore, what is the correlation between the FI-Lab and prognosis in older AECOPD inpatients? Our objective is to study the prognostic value of the FI-Lab in older Chinese patients who were admitted because of AECOPD.

\section{Methods}

\section{Study Design and Study Participants}

A retrospective observational study was conducted at Zhenjiang First People's Hospital, China, between September 2016 and June 2019. This is a tertiary teaching hospital in China. The study protocol was approved by the hospital ethics committee. All data were collected retrospectively from the hospital database. Because of the retrospective characteristic of the study design, the patient consent to review their medical records was waived by the ethics committee. The authors complied strictly with the Declaration of Helsinki and covered patient data confidentiality.

The primary clinical diagnosis of AECOPD patients was at least 60 years of age. In total, 1932 patients (1824 survived and 108 died in hospital) were screened for this study. We excluded inpatients with secondary causes such as lung cancer, bronchiectasis, asthma, interstitial lung disease and active pulmonary tuberculosis. Each patient was admitted to the study only once during his or her initial hospitalization. Patients with incomplete data (clinical data, auxiliary examination, hematologic examination, etc.) were excluded. A total of 285 were excluded due to missing data, of which 61 were excluded due to missing data on FI-Lab tool. We also excluded patients who were automatically discharged or moved to another hospital.

After that exclusion, there were 426 survivors and 98 survivors. Previous differences in the characteristics of survivors and non-survivors may lead to biased estimates. To reduce this bias, we used propensity score matching (PSM) techniques. ${ }^{22}$ A logistical regression model was used to estimate the propensity to participate in both groups according to a set of observed covariates. PSM matching (1:1 matching) covariates included: age, gender, the number of smokers, the number of drinkers, history of comorbid diseases (including type 2 diabetes, hypertension, myocardial infarction, or stroke). Finally, 77 survivors and 77 non-survivors were included in the study. The flow chart of subject inclusion is summarized in Figure 1. 


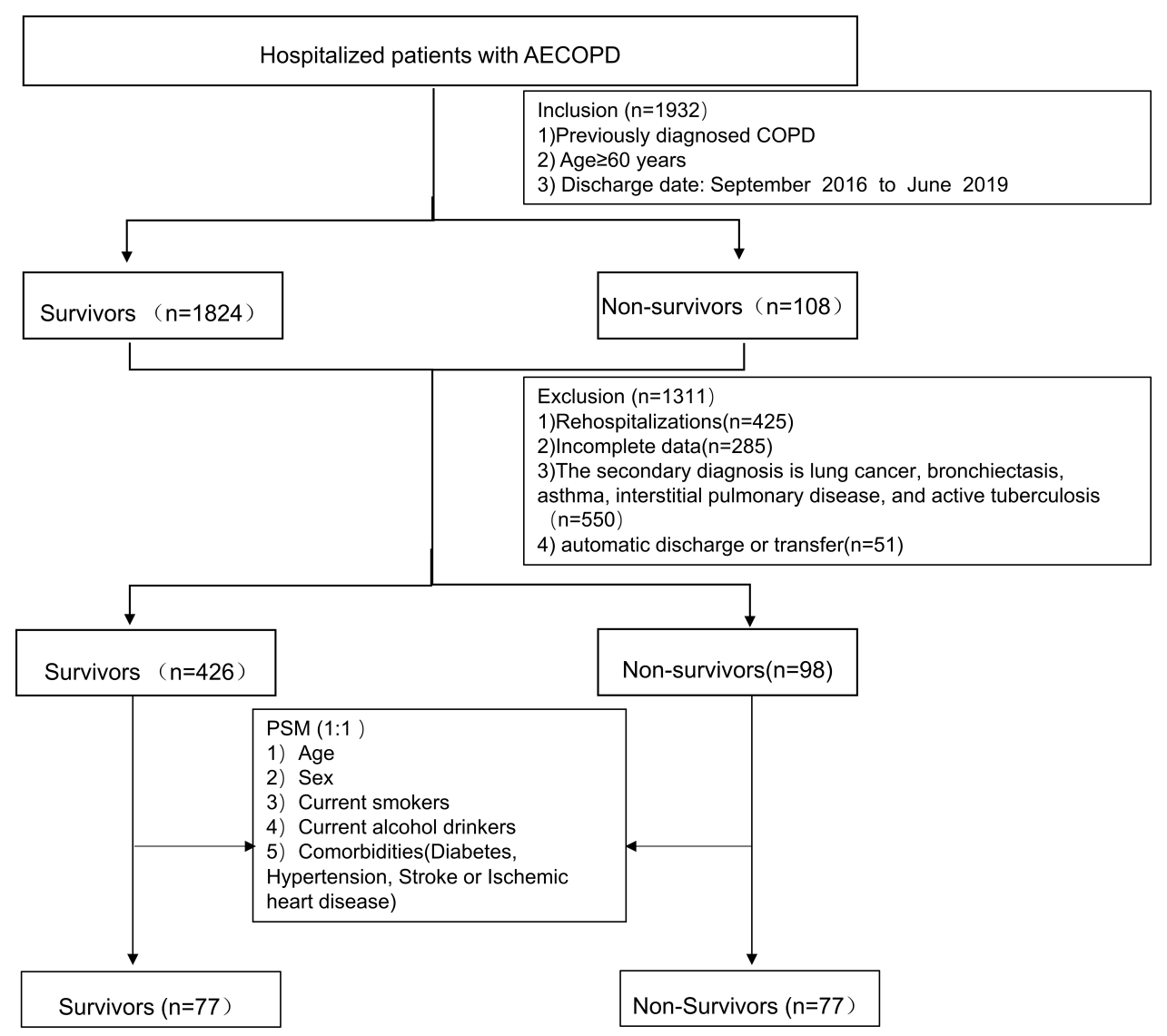

Figure I Flow chart of patient admission.

\section{Definitions COPD and AECOPD}

The COPD diagnosis was established by a consistent airflow obstruction on spirometry (FEV1/forced vital capacity $<0.70$ ). ${ }^{23}$ The exacerbation of COPD (AECOPD)was defined as an acute change in a patient's respiratory symptoms that is beyond normal variability and is sufficient to warrant a change in therapy. ${ }^{23}$

\section{FI-Lab}

Rockwood et $\mathrm{al}^{19}$ and Howlett et $\mathrm{al}^{20}$ developed an FI (the FI-Lab) of up to 23 variables based on 21 routine blood tests plus measured systolic and diastolic blood pressure based on deficit accumulation (Supplementary Table 1). The FI-Lab can meet several criteria of ideal biomarkers (influential to the pathogenesis, easy to measure, sensitive to changes, improved to an intervention, prognostic to outcome) provided by Mcshane et $\mathrm{al}^{24}$ and Gruttola et al. ${ }^{25}$ FI-Lab has been built by evaluating each variable as 0 or 1 . ' 0 ' indicates that values are within the normal cut-offs but ' 1 ' indicates that values are outside the normal cut-offs as deficits. An FILab score is built by counting the number of deficits and dividing by the total number of items tested for a score between 0 and 1 . For example, a patient with deficits in six variables of the 23-item FI-Lab would have an FI-Lab value of 0.26 ( 6 divided by 23). A higher score indicates greater frailty.

An FI-Lab score was calculated only if over $70 \%$ of the variables (items 16 to 23 ) were available. We treated the FILab score as a continuous variable based on previous studies and ranked participants based on the FI-Lab value in four categories: $<0.2,0.2-0.4,0.4-0.6$, and $>0.6$. We have also tried to find an optimal threshold for FI-Lab to predict mortality.

\section{DECAF Score}

DECAF consists of five parameters: dyspnea (D), eosinopenia (E), consolidation (C), acidemia (A), and atrial fibrillation (F). Evaluating DECAF in AECOPD patients can help to predict mortality. DECAF is a predictor of AECOPD with a score range between 1 and 6 . A higher score indicates the poorer condition. A recent study found that patients with a DECAF score of four or more are at 
high risk of mortality. ${ }^{6}$ Based on the DECAF score, we ranked participants at four levels: $\leq 2,3,4$, and $\geq 5$.

\section{Measurements}

Electronic Data were collected from the inpatient hospital database. Patients characteristics such as gender, age, current smokers, current alcohol drinkers, comorbid diseases (diabetes, hypertension, myocardial infarction or stroke), length of stay, and rehospitalization were documented. Initial blood results and systolic pressure, diastolic pressure were extracted on admission.

Blood tests include complete blood count (total leukocyte, neutrophil, eosinophil, lymphocyte, platelet counts, mean platelet volume, red cell distribution width, hemoglobin); blood biochemical (total protein, albumin, aspartate aminotransferase, calcium, creatinine, urea, fasting blood glucose, alkaline phosphatase, phosphorus, potassium, sodium); thyroid function (thyroid-stimulating hormone, thyroxine, free thyroxine); syphilis; hematopoietic raw materials (serum folate, vitamin $\mathrm{B} 12)$; inflammatory markers including C-reactive protein(CRP), neutrophil-to-lymphocyte ratio(NLR), platelet-tolymphocyte ratio(PLR); blood gas analysis.

\section{Statistical Analysis}

Data were analyzed using IBM SPSS for Windows, Version 23.0 (IBM Corp, Armonk, NY). The survivors were compared to the non-survivors. The baseline difference between the groups was matched by PSM. The median with interquartile range was employed for nonparametric continuous variables, and the mean \pm standard deviation was used for parametric continuous variables. Count and percentage were used when applicable. Mann-Whitney $U$-tests for nonparametric continuous variables or Student's t-tests for parametric continuous variables. Chi-square tests were employed for dichotomous variables. A multivariate logistic regression analysis was used to identify prognostic factors for hospital mortality. The area under the receiver operator characteristic curve (AUCs) was used to assess the performance of FI-Lab and DECAF in hospital mortality prediction. The comparison of the AUCs was performed using the DeLong method. ${ }^{26}$ The Youden Index method was used to determine the optimal thresholds of FI-Lab or DECAF to predict mortality. A two-tailed p-value of 0.05 was considered statistically significant.

\section{Results}

\section{General Characteristics of Survivors and Non-Survivors on Admission}

We reviewed the case records for 34 months. Finally, according to the strict inclusion and exclusion criteria and PSM matching method, a total of 154 patients were enrolled, including 77 survivors and 77 non-survivors independently (Figure 1). The general characteristics in two groups were shown in Table 1. The mean age of these 154 patients was $79.73 \pm 8.38$ years. Men were more common in both groups. 85 patients $(55.19 \%)$ were current smokers while only 21 patients $(13.64 \%)$ were current alcohol drinkers. Among the comorbidities, hypertension is the most common (56.49\%). There were no statistically significant difference in blood pressure between the two groups.

Table I The Balanced General Characteristics of Survivors and Non-Survivors on Admission

\begin{tabular}{|l|c|c|c|}
\hline Indexes & Survivors (n=77) & Non-Survivors (n=77) & P-value \\
\hline Age (years) & $79.38 \pm 8.14$ & $80.09 \pm 8.65$ & 0.207 \\
\hline Men (\%) & $57(74.0)$ & $52(67.5)$ & 0.376 \\
\hline Current smokers (\%) & $45(58.4)$ & $40(51.9)$ & 0.418 \\
\hline Current alcohol drinkers (\%) & $11(14.3)$ & $10(13)$ & 0.814 \\
\hline Length of hospital days & $10.06 \pm 4.53$ & $10.26 \pm 8.70$ & 0.862 \\
\hline Comorbidities (\%) & $12(15.6)$ & $16(20.8)$ & 0.664 \\
Diabetes & $41(53.2)$ & $46(59.7)$ & 0.416 \\
Hypertension & $17(22.1)$ & $26(33.8)$ & 0.106 \\
Stroke or Ischemic heart disease & & & \\
\hline Blood pressure & $136.65 \pm 22.63$ & $131.18 \pm 22.64$ & 0.136 \\
Systolic blood pressure (mmHg) & $77.92 \pm 11.52$ & $75.61 \pm 15.00$ & 0.285 \\
Diastolic blood pressure (mmHg) & & & \\
\hline
\end{tabular}




\section{Some Laboratory Findings of Survivors and Non-Survivors}

According to previous researches, complete blood count results, inflammatory indicators, partial pressure of carbon dioxide $\left(\mathrm{PaCO}_{2}\right)$ are always associated with AECOPD. The above laboratory findings of survivors and nonsurvivors are shown in Table 2. In the non-survivors, lymphocyte counts, eosinophil counts, hemoglobin, platelet count level were significantly lower, whereas leukocyte counts, neutrophil counts, red blood cell distribution (RDW) level were significantly higher compared with the survivors (all $P<0.05$ ). There were no significant differences in other parameters. We also found non-survivors had higher partial pressure of carbon dioxide $\left(\mathrm{PaCO}_{2}\right)$ and inflammatory markers (NLR, PLR, CRP).

\section{FI-Lab and DECAF of Survivors and Non-Survivors}

We calculated FI-Lab for all patients. FI-Lab values were divided into four categories: $<0.2,0.2-0.4,0.4-0.6$, and $>0.6$ and the FI-Lab of $0.2-0.39$ was most common $(\mathrm{n}=64 ; 41.56 \%)$. Among survivors, 68 patients $(88.3 \%)$ had FI-Lab values of $<0.4$ and the most common were 0.2 to 0.39 ( $\mathrm{n}=32 ; 41.56 \%)$. In non-survives, 58 patients (75.3\%) had FI-Lab values of $>0.4$ and the most common values were $0.4-0.6(n=39 ; 50.65 \%)$. The mean FI-Lab of non-survives was statistically higher to that of survivors $(0.51 \pm 0.13$ versus $0.29 \pm 0.10, P=0.000)$ (Table 3$)$.

We also calculated DECAF scores for all patients. DECAF scores were ranked in four categories: $\leq 2,3,4$, and $\geq 5$. A DECAF score of 4 was most prevalent ( $n=53$; $34.41 \%)$. The most common DECAF scores in nonsurvivors were $\geq 4(\mathrm{n}=71,92.21 \%)$ while the scores were $<4$ in survivors $(n=56 ; 72.73 \%)$. The mean DECAF score of non-survivors was statistically higher than that of survivors (4.45 \pm 0.80 versus $3.03 \pm 0.90, P=0.000)$, as shown in Table 3.

\section{Multivariate Logistic Regression Analysis}

Some complete blood count parameters (leukocytes, neutrophils, hemoglobin, et al) were already included in the blood parameters of 21 FI-Lab items and were no longer included in the logistic regression equation. Variables (age, gender, blood pressure, comorbidities, platelet count, et al) not statistically significant were also not included in the logistic regression equation. Finally, eight statistically significant parameters (all $P<0.05$ ) were included in the logistic regression model, including CRP, NLR, PLR, DECAF rank, FI-Lab rank, $\mathrm{PaCO}_{2}$, RDW, eosinophil count in the logistic regression model. The results showed that the DECAF and FI-Lab ranks correlated with hospital mortality in AECOPD patients $(P=0.000)$, as shown in Table 4.

Table 2 Some Laboratory Findings of Survivors and Non-Survivors

\begin{tabular}{|c|c|c|c|}
\hline Parameters & Survivors $(n=77)$ & Non-Survivors $(n=77)$ & $P$-value \\
\hline \multicolumn{4}{|c|}{ Complete blood count results } \\
\hline Leukocyte count $\left(\times 10^{9} / \mathrm{L}\right)$ & $7.66 \pm 3.87$ & $10.70 \pm 7.40$ & $0.002 *$ \\
\hline Neutrophil count $\left(\times 10^{9} / \mathrm{L}\right)$ & $6.14 \pm 3.59$ & $9.36 \pm 7.08$ & $0.001 *$ \\
\hline Lymphocyte count $\left(\times 10^{9} / \mathrm{L}\right)$ & $1.14 \pm 0.78$ & $0.84 \pm 0.72$ & $0.016 *$ \\
\hline Eosinophil count $\left(\times 10^{9} / \mathrm{L}\right)$ & $0.02(0.00,0.10)$ & $0(0,0.01)$ & $0.003 *$ \\
\hline Platelet count $\left(\times 10^{9} / \mathrm{L}\right)$ & $197.58 \pm 73.24$ & $185.27 \pm 86.34$ & 0.341 \\
\hline $\mathrm{MPV}(\mathrm{fl})$ & $10.31 \pm 1.32$ & $10.29 \pm 1.26$ & 0.906 \\
\hline RDW (\%) & $13.74 \pm 1.06$ & $15.36 \pm 2.89$ & $0.000 *$ \\
\hline Hemoglobin $(g / l)$ & $125.19 \pm 16.90$ & $107.90 \pm 23.46$ & $0.000 *$ \\
\hline \multicolumn{4}{|l|}{ Inflammatory markers } \\
\hline NLR & $5.36(3.27,10.29)$ & II.60(6.20, 19.35) & $0.000 *$ \\
\hline PLR & $191.25(\mid 32.20,288.27)$ & $245.0(147.9,406.65)$ & $0.047 *$ \\
\hline $\mathrm{CRP}(\mathrm{mg} / \mathrm{l})$ & $13.17(4.08,59.35)$ & $25.24(9.90,79.45)$ & $0.011 *$ \\
\hline \multicolumn{4}{|l|}{ Blood gas analysis } \\
\hline $\mathrm{PaCO}_{2}(\mathrm{mmHg})$ & $51.13 \pm 13.48$ & $64.49 \pm 21.63$ & $0.000 *$ \\
\hline
\end{tabular}

Note: $* P$-value $<0.05$.

Abbreviations: NLR, neutrophil and lymphocyte ratio; RDW, red blood cell distribution; MPV, mean platelet volume; CRP, C-reactive protein; NLR, neutrophil-tolymphocyte ratio; PLR, platelet-to-lymphocyte ratio; $\mathrm{PaCO}_{2}$, partial pressure of carbon dioxide. 
Table $3 \mathrm{FI}$-Lab and DECAF of Survivors and Non-Survivors at the Time of Admission

\begin{tabular}{|l|c|c|c|}
\hline Values & $\begin{array}{c}\text { Survivors } \\
(\mathbf{n}=\mathbf{7 7})\end{array}$ & $\begin{array}{c}\text { Non-Survivors } \\
\text { (n=77) }\end{array}$ & P-value \\
\hline $\begin{array}{l}\text { DECAF Rank } \\
\text { (n, \%) } \\
\leq 2\end{array}$ & 24 & 1 & $0.000^{*}$ \\
3 & 32 & 5 & $0.000^{*}$ \\
4 & 17 & 36 & $0.000^{*}$ \\
$\geq 5$ & 4 & 35 & $0.000^{*}$ \\
\hline $\begin{array}{l}\text { Mean DECAF } \\
\text { score }\end{array}$ & $3.03 \pm 0.90$ & $4.45 \pm 0.80$ & $0.000^{*}$ \\
\hline FI-Lab Rank & & & \\
(n, \%) & & & $0.000^{*}$ \\
$<0.2$ & $20(25.97)$ & 18 & $0.000^{*}$ \\
$0.20-0.39$ & $48(62.34)$ & 39 & $0.000^{*}$ \\
$0.40-0.6$ & $9(11.68)$ & 19 & $0.000^{*}$ \\
$\geq 0.60$ & 1 & $0.51 \pm 0.13$ & $0.000^{*}$ \\
\hline Mean FI-Lab & $0.29 \pm 0.10$ & & \\
\hline
\end{tabular}

Note: $* P$-value $<0.05$.

\section{ROC Analysis}

ROC curves were calculated to estimate FI-Lab and DECAF concerning mortality (Figure 2). The AUCs were 0.906 for FI-Lab and 0.870 for DECAF ( $P=0.2991$ ). When the FI-Lab was 0.4388 , the sensitivity, specificity, and Youden index were $70.1 \%, 96.1 \%$, and 0.675 respectively. When the DECAF score was 3.5 , sensitivity, specificity, and Youden index were $92.2 \%, 72.7 \%$, and 0.649 . FI-Lab has a slightly stronger screening ability than DECAF.

\section{Discussion}

We explored the prognostic value of the FI-Lab in AECOPD inpatients in China. Rather than using the traditional respiratory indices to predict mortality in AECOPD, we used the interdisciplinary FI-Lab to predict risk among patients with AECOPD. Our study demonstrated that the mean FI-Lab values and mean DECAF scores in nonsurvivors were much higher than those of survivors. Inpatient mortality increased in accordance with increases

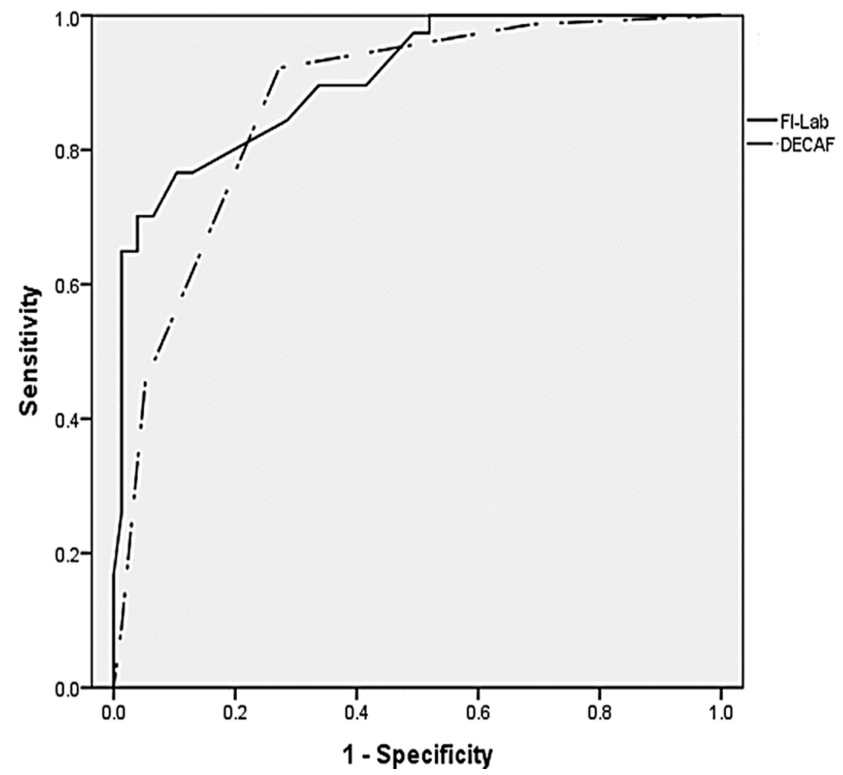

Figure 2 ROC curves for FI-Lab and DECAF.

in the FI-Lab or DECAF scores. Therefore, both the FILab and DECAF scores can be successfully applied to predict in-hospital mortality.

This study found that single indexes, including RDW, CRP, and partial pressure of carbon dioxide $\left(\mathrm{PaCO}_{2}\right)$, in non-survivors were significantly higher than those of survivors. Moreover, eosinophil counts in non-survivors were significantly lower than those of survivors. However, after logistic regression analysis, only the FI-Lab rank and DECAF rank were risk factors for death. This suggests that single indexes show low prognostic value. Gu et $\mathrm{al}^{27}$ pointed out that no single biomarkers can be effectively applied to the quantitative diagnosis and prognosis of AECOPD. Therefore, we should seek new comprehensive biomarkers such as FI-Lab and DECAF scoring.

Nafae et $\mathrm{al}^{28}$ reported that DECAF scores were significant predictors of hospital mortality, with areas under the curve (AUCs) of 0.870 . The AUCs of the DECAF scores in our study were occasionally 0.870 . Many studies ${ }^{5,12}$ have shown that the value of DECAF scores in predicting mortality in AECOPD patients is superior to other respiratory scoring systems, including the acute physiology and

Table 4 Results of Multivariate Logistic Analysis (Forward Stepwise)

\begin{tabular}{|l|c|c|c|c|c|c|}
\hline Variables & $\boldsymbol{\beta}$ & Sb & Wald $\chi \mathbf{2}$ & Odds Ratio & 95\% Cl: Lower-Upper Limit & $\boldsymbol{P}$-value \\
\hline FI-Lab Rank & 2.164 & 0.444 & 23.753 & 8.705 & $3.646-20.782$ & $0.000 *$ \\
DECAF Rank & 1.726 & 0.354 & 24.748 & 5.620 & $2.811-11.236$ & $0.000 *$ \\
\hline
\end{tabular}

Note: $* P$-value $<0.05$. 
chronic health evaluation (APACHE) score, the community-acquired pneumonias (CAPS) score, the confusion, urea nitrogen, respiratory rate, and blood pressure, 65 years of age and older (CURB-65) score, and the blood urea nitrogen, altered mental status, and pulse, 65 years of age and older (BAP-65) score. However, the DECAF scoring has some weaknesses. First, our study suggested that DECAF scores had high sensitivity but low specificity (when the DECAF cutoff score was 3.5, the sensitivity and specificity were $92.2 \%$ and $72.7 \%$, respectively, and the Youden index was 0.649). One study even showed that the sensitivity of the DECAF score for predicting mortality in AECOPD patients was $100 \%$, but the specificity was poor $(34.1 \%){ }^{29}$ Second, the DECAF score consists of five parameters and covers clinical, serological, radiological, and electrocardiography scales. Therefore, this score is difficult to assess and inconvenient for repeated testing, because imaging changes are relatively slow in patients. Finally, pulmonary consolidation of imaging tests often suggests pneumonia, and there is a debate with regard to judging whether AECOPD includes AECOPD with pneumonia.

The association between frailty and mortality has been well established, ${ }^{15}$ based on the fact that COPD and frailty share a common pathogenesis and that co-morbidity is more prevalent in older patients. ${ }^{30,31}$ The FI-Lab used in this paper is a 23 -item indicator (21 hematological indices plus systolic and diastolic blood pressures) that was proposed by Howlett et al. ${ }^{19,20,32}$ The FI-Lab has been widely used for objective assessments of the degrees of frailty. FILab values were found to be significantly higher in nonsurvivors than in survivors. The FI-Lab was significantly correlated with mortality, with an AUC area of 0.906, suggesting that the predictive value of the FI-Lab is slightly more reliable than that of the DECAF scoring (AUCs of 0.870). FI-Lab assessments are objective, easy to apply at the bedside, and convenient to repeat. When the FI-Lab was 0.4388 , the specificity for predicting mortality was $96.1 \%$, which was much higher than with the DECAF scores. However, although the specificity of the FI-Lab reached $96.1 \%$, its sensitivity was relatively poor (only $70.1 \%$ ). Therefore, we still need to find a better predictor through future research.

\section{Limitations}

This study has some limitations. First, this was a retrospective study, we need a prospective cohort study to confirm the conclusion. Second, although we screened numerous cases (total cases were 1932), the final sample size was relatively small $(n=154)$. Strict exclusion criteria and excluded patients with missing data may lead to selection bias and reporting bias independently. Third, because this was a retrospective study, it was not able to analyze some critical outcomes in detail including causes of death, falls during hospitalization, quality of life, the incidence of acute cardiovascular events, and social support. Finally, it may take time to determine abnormalities in the 23 elements. If the FI-Lab can be coded into a program (or an App) and integrated into hospital information systems (HIS), it can be calculated automatically.

\section{Conclusion}

This study demonstrates that an FI constructed from routinely collected laboratory and clinical data identifies older AECOPD adults at increased risk of death. FI-Lab is a simple, effective, and objective indicator that can quickly help clinicians stratify AECOPD patients. FI-Lab dynamic monitoring can be of great clinical value in understanding patient progress and predicting risk of death for AECOPD patients.

\section{Acknowledgments}

The project was supported by a research grant by the Hospital-level Scientific Fund of Zhenjiang First People's Hospital (Y2019006).

\section{Disclosure}

All the authors declare no conflicts of interest in this work.

\section{References}

1. Woodruff PG, Agusti A, Roche N, Singh D, Martinez FJ. Current concepts in targeting chronic obstructive pulmonary disease pharmacotherapy: making progress towards personalised management. Lancet. 2015;385(9979):1789-1798. doi:10.1016/S0140-6736(15) 60693-6

2. Tamer A, Baess AI, Monsif DAA, Elnasharty AAA. Role of C-reactive protein and interleukin-6 in predicting the prognosis of ICU-admitted patients with acute exacerbation of COPD. Egypt $J$ Chest Dis Tuberculosis. 2014;63(4):829-835. doi:10.1016/j. ejcdt.2014.06.003

3. Duman D, Aksoy E, Agca MC, et al. The utility of inflammatory markers to predict readmissions and mortality in COPD cases with or without eosinophilia. Int $J$ Chron Obstruct Pulmon Dis. 2015;10:2469-2478. doi:10.2147/COPD.S90330

4. Leuzzi G, Galeone C, Taverna F, Suatoni P, Morelli D, Pastorino U. C-reactive protein level predicts mortality in COPD: a systematic review and meta-analysis. Eur Respir Rev. 2017;26(143):160070. doi:10.1183/16000617.0070-2016

5. Steer J, Gibson J, Bourke SC. The DECAF Score: predicting hospital mortality in exacerbations of chronic obstructive pulmonary disease. Thorax. 2012;67(11):970-976. doi:10.1136/thoraxjnl-2012-202103 
6. Memon MA, Faryal S, Brohi N, Kumar B. Role of the DECAF score in predicting in-hospital mortality in acute exacerbation of chronic obstructive pulmonary disease. Cureus. 2019;11(6):e4826. doi: $10.7759 /$ cureus. 4826

7. Surapaneni KP. S, N S. DECAF score for assessing prognosis in Acute Exacerbation of COPD. J Assoc Physicians India. 2020;68 (1):82.

8. Rahimirad S, Ghafari M, Ansarin K, Rashidi F, Rahimi-Rad MH. Elevated red blood cell distribution width predicts mortality in acute exacerbation of COPD. Pneumologia. 2016;65(2):85-89.

9. Epstein D, Nasser R, Mashiach T, Azzam ZS, Berger G. Increased red cell distribution width: a novel predictor of adverse outcome in patients hospitalized due to acute exacerbation of chronic obstructive pulmonary disease. Respir Med. 2018;136:1-7. doi:10.1016/j. rmed.2018.01.011

10. Wu CW, Lan CC, Hsieh PC, Tzeng IS, Wu YK. Role of peripheral eosinophilia in acute exacerbation of chronic obstructive pulmonary disease. World J Clin Cases. 2020;8(13):2727-2737. doi:10.12998/ wjcc.v8.i13.2727

11. Yao C, Liu X, Tang Z. Prognostic role of neutrophil-lymphocyte ratio and platelet-lymphocyte ratio for hospital mortality in patients with AECOPD. Int J Chron Obstruct Pulmon Dis. 2017;12:2285-2290. doi: $10.2147 / C O P D . S 141760$

12. Huang Q, He C, Xiong H, et al. DECAF score as a mortality predictor for acute exacerbation of chronic obstructive pulmonary disease: a systematic review and meta-analysis. BMJ Open. 2020;10 (10):e037923. doi:10.1136/bmjopen-2020-037923

13. Rockwood K, et al. A global clinical measure of fitness and frailty in elderly people. Cmaj Canadian Medical Association Journal. 2005;173(5):489-495. doi:10.1503/cmaj.050051

14. Kizilarslanoglu MC, Civelek R, Kilic MK, et al. Is frailty a prognostic factor for critically ill elderly patients. Aging Clin Exp Res. 2017;29(2):1-9.

15. Morley JE, Vellas B, van Kan GA, et al. Frailty consensus: a call to action. J Am Med Dir Assoc. 2013;14(6):392-397. doi:10.1016/j. jamda.2013.03.022

16. Fragoso CAV, Enright PL, McAvay G, Van Ness PH, Gill TM. Frailty and respiratory impairment in older persons. Am J Med. 2012;125 (1):1-86.

17. Limpawattana $\mathrm{P}$, Putraveephong S, Inthasuwan $\mathrm{P}$, Boonsawat W, Theerakulpisut D, Chindaprasirt J. Frailty syndrome in ambulatory patients with COPD. Int $J$ Chron Obstruct Pulmon Dis. 2017;12:1193-1198. doi:10.2147/COPD.S134233

18. Searle SD, Mitnitski A, Gahbauer EA, Gill TM, Rockwood K. A standard procedure for creating a frailty index. BMC Geriatr. 2008;8:24. doi:10.1186/1471-2318-8-24

19. Rockwood K, McMillan M, Mitnitski A, Howlett SE, Frailty Index A. Based on common laboratory tests in comparison with a clinical frailty index for older adults in long-term care facilities. $J$ Am Med Dir Assoc. 2015;16(10):842-847. doi:10.1016/j.jamda. 2015.03.027
20. Howlett SE, Rockwood MR, Mitnitski A, Rockwood K. Standard laboratory tests to identify older adults at increased risk of death. BMC Med. 2014;12(1):171. doi:10.1186/s12916-014-0171-9

21. Yang M, Zhuo Y, Hu X, Xie L. Predictive validity of two frailty tools for mortality in Chinese nursing home residents: frailty index based on common laboratory tests (FI-Lab) versus FRAIL-NH. Aging Clin Exp Res. 2018;30(12):1445-1452. doi:10.1007/s40520-018-1041-7

22. Rosenbaum PR, Rubin DB. The central role of the propensity score in observational studies for causal effects. Biometrika. 1983;70 (1):41-55. doi:10.1093/biomet/70.1.41

23. Vogelmeier CF, Criner GJ, Martinez FJ, et al. Global strategy for the diagnosis, management and prevention of chronic obstructive lung disease 2017 report: GOLD executive summary. Respirology. 2017;22(3):575-601.

24. Mcshane LM, Altman DG, Willi S. Identification of clinically useful cancer prognostic factors: what are we missing. J Natl Cancer Inst. 2005;97(14):1023-1025. doi:10.1093/jnci/dji193

25. Gruttola VGD, Clax P, Demets DL, et al. Considerations in the evaluation of surrogate endpoints in clinical trials: summary of a national institutes of health workshop. $J$ Natl Cancer Inst. 2001;22(5):485-502.

26. DeLong ER, DeLong DM, Clarke-Pearson DL. Comparing the areas under two or more correlated receiver operating characteristic curves: a nonparametric approach. Biometrics. 1988;44(3):837-845. doi: $10.2307 / 2531595$

27. Gu Y. [Biomarker in acute exacerbation of chronic obstructive pulmonary disease]. Zhonghua Jie He Hu Xi Za Zhi. 2014;37 (4):247-249. Chinese.

28. Nafae R, Embarak S, Gad DM. Value of the DECAF score in predicting hospital mortality in patients with acute exacerbation of chronic obstructive pulmonary disease admitted to Zagazig University Hospitals, Egypt. Egypt $J$ Chest Dis Tuberculosis. 2015;64(1):35-40. doi:10.1016/j.ejcdt.2014.10.007

29. Sangwan V, Chaudhry D, Malik R. Dyspnea, eosinopenia, consolidation, acidemia and atrial fibrillation score and BAP-65 score, tools for prediction of mortality in acute exacerbations of chronic obstructive pulmonary disease: a comparative pilot study. Indian J Crit Care Med. 2017;21(10):671-677. doi:10.4103/ijccm.IJCCM_148_17

30. Gale NS, Albarrati AM, Munnery MM, et al. Frailty: a global measure of the multisystem impact of COPD. Chron Respir Dis. 2018;15 (4):347-355. doi:10.1177/1479972317752763

31. Bernabeu-Mora R, Oliveira-Sousa SL, Piedad Sánchez-Martínez M, et al. Frailty transitions and associated clinical outcomes in patients with stable COPD: a longitudinal study. PLoS One. 2020;15(4): e0230116. doi:10.1371/journal.pone.0230116

32. Blodgett JM, Theou O, Howlett SE, Wu FCW, Rockwood K. A frailty index based on laboratory deficits in community-dwelling men predicted their risk of adverse health outcomes. Age Ageing. 2016;45(4):afw054. doi:10.1093/ageing/afw054

\section{Publish your work in this journal}

The International Journal of COPD is an international, peer-reviewed journal of therapeutics and pharmacology focusing on concise rapid reporting of clinical studies and reviews in COPD. Special focus is given to the pathophysiological processes underlying the disease, intervention programs, patient focused education, and self management protocols. This journal is indexed on PubMed Central, MedLine and CAS. The manuscript management system is completely online and includes a very quick and fair peer-review system, which is all easy to use. Visit http://www.dovepress.com/testimonials.php to read real quotes from published authors. 